\title{
CARACTERÍSTICAS EPIDEMIOLÓGICAS DE ACCIDENTES LABORALES
}

\author{
EN EL HOSPITAL ANTONIO LORENA. 2010 - 2014
}

José Rafael Ugarte Martínez ${ }^{1}$, Liliana Erikha Ferro Galdos².

\section{RESUMEN}

Las características epidemiológicas de los accidentes laborales en el hospital Antonio Lorena, tiene por objeto determinar si la edad, tiempo de servicios, grupo ocupacional y grado de instrucción tienen relación con la forma de producción de los accidentes laborales ocurridos durante las horas de trabajo entre los años 2010 y 2014 . Actualmente no se dispone de información en el hospital sobre los accidentes en sus trabajadores. Se revisaron 91 formatos de accidentes laborales en la unidad de epidemiología del hospital. Se llenó la ficha de recolección de datos, luego se pusieron los datos al programa Excel y SPSS 20. Es un estudio no experimental, transversal, retrospectivo y correlacional. El promedio de la edad de los trabajadores accidentados es de 37,44 años de una total de 712 trabajadores, el tiempo de servicios promedio es de 9,41 años de trabajo y el grupo de

1 Magíster en Medicina Humana. Médicos asistentes del hospital Antonio Lorena. E-mail: umar21@hotmail.com

2 Médico Cirujano. 
trabajadores con menos de 5 años de servicio fueron los más lesionados con 54 casos. El grupo ocupacional más afectado fueron las enfermeras con 31 accidentados. La forma de los accidentes con mayor frecuencia fueron los pinchazos con el $67 \%$ de los casos. En cuanto al nivel de correlación se usó el test de Spearman, debido a que el test de Kolmogorov Smirnov resulto no significativo para normalidad $(2,697$ $\mathrm{p}<0,001)$. Se observó mediana correlación entre edad del trabajador, tiempo de servicios con la forma del accidente laboral $(\mathrm{rho}=-0,516 \mathrm{p}<0,001$ y rho $=-0,490 \mathrm{p}<0,001)$, ninguna correlación entre el nivel de educación del trabajador y la forma del accidente laboral $(\mathrm{rho}=-0,086 \mathrm{p}=0,420)$.

PALABRAS CLAVE: Accidentes, laborales, características, epidemiológicas, hospital, herida, punzante.

\section{ABSTRACT}

The epidemiological features of occupational injuries of Antonio Lorena's hospital find to determinate if the age, work time. Occupational group and level of education have relationship with kind of injuries made during working between 2010 and 2014. Actually, we don't know about occupational injuries. I reviewed 91 occupational injuries in epidemiological office, then i filled epidemiological registration form and features were analyzed in Excel and SPSS 20. This is a non-experimental, retrospective, one view and correlational study. The average of injured workers was 37,44 years old of 643 total workers, worked time average was 9,41 years and workers with less than 5 years in hospital were more injured with 54 cases. Nurses were more affected with 31 cases. Needle injuries were $67 \%$. Correlation level was determined with Spearman test because distribution of cases was non-parametric tested with Kogomorov Smirnov test $(2,697 \mathrm{p}<0,001)$. It saw middle correlation between workers age, worked time with kind of occupational injuries $(\mathrm{rho}=-0,516 \mathrm{p}<0,001$ y rho $=-0,490 \mathrm{p}<0,001)$, non-correlation between educational level and kind of occupational injuries (rho $=$ $-0,086 \mathrm{p}=0,420$ ).

KEYWORDS: Occupational, injuries, features, epidemiological, hospital, wound, needle.

$\mathrm{E}$ tema de estudio corresponde a la línea de investigación de la calidad de las prestaciones de salud y permite conocer el comportamiento de las características epidemiológicas de los accidentes laborales y a su vez poder realizar sugerencias para disminuir los mencionados accidentes.

El encapuchado de la aguja es la principal causa de los accidentes punzantes, se considera inadecuada (1). También se consideran otros factores en la producción de los accidentes laborales los factores ambientales, como las condiciones de trabajo; factores personales, relacionado con el comportamiento del trabajador; factores mecánicos relacionados con la práctica del uso de las agujas (2 y 3 ).
En Albacete España se encontró que el grupo de edad con más accidentes laborales fue el de 30 a 39 años, seguidos de 20 a 29 años y más en mujeres que en varones y los grupos ocupacionales más lesionados fueron las enfermeras y los técnicos de enfermería (5). En contraposición de lo reportado en el hospital universitario de San Carlos, Madrid España en donde la edad promedio de accidentados era de 61 años, mencionaron también un alto porcentaje de omisiones (6). En Valencia en el hospital de Agudos refieren que la mayor parte de los accidentes se produjeron en la tarde, con el aumento de la edad y en cocina y lavandería, siendo los accidentes más frecuentes los esguinces y las tendinitis (7).

En Sao Paulo Brasil publicaron que los factores asociados a los accidentes laborales fueron la sobrecarga de trabajo, mala calidad de los materiales, agresividad de los clientes y falta de atención en el encapuchado de las agujas (9). En Montevideo Uruguay, los accidentes más frecuentes fueron por punción 48,5\%, seguido por traumatismos con $17,1 \%$ y el grupo más afecto fueron los técnicos de enfermería (11).

En el hospital Alberto Sabogal de Essalud Lima, aplicando encuestas tuvo como resultados que al menos $21,6 \%$ tuvo un accidente laboral, de los cuales el $86.1 \%$ fue punzo cortante y $13.8 \%$ de exposición mucocutánea. Produciéndose la mayoría de los accidentes en el turno de la mañana, el segmento lesionado fueron los dedos de la mano.

El problema central del estudio es conocer el comportamiento las características epidemiológicas de los accidentes laborales del hospital Antonio Lorena del Cusco durante los años 2010 al 2014, teniendo como objetivo general el determinar las características epidemiológicas de los accidentes laborales en el Hospital Antonio Lorena 
del Cusco 2010 - 2014. Los objetivos específicos son: Analizar el grupo de edad con mayor cantidad de accidentes laborales; cuantificar el tiempo de servicios de los trabajadores con accidentes laborales; identificar el grupo laboral con más accidentes laborales; conocer el grado de instrucción de los trabajadores con accidentes laborales y analizar el tipo de accidente laboral más frecuente.

El estudio se realiza en un contexto en el que no se conoce en el hospital Antonio Lorena el comportamiento de las variables de los trabajadores que reportaron accidentes laborales, siendo la unidad de análisis los informes de los accidentes laborales de los trabajadores lesionados. Al conocer los resultados nos permitirá plantear sugerencias para disminuir los accidentes laborales dentro del hospital Antonio Lorena.

\section{METODOLOGIA}

El diseño del estudio es no experimental porque no manipulamos deliberadamente las variables (22). Es transversal, porque es una observación en el tiempo $(19,20$, 21, 22). Dentro de los estudios no experimentales, transversales, pertenece al tipo correlacional/causal porque observa dos variables y ver si se relacionan en un momento determinado.

Es retrospectivo porque se realiza las observaciones de sucesos ocurridos en los años pasados 2010 al 2014, de los trabajadores accidentados en el Hospital Antonio Lorena.

La población son todos los trabajadores del Hospital Antonio Lorena y la muestra es de tipo no probabilístico, porque depende de las causas relacionadas con las características que hace la muestra. Es un muestreo por sujeto tipo $(22,23,24)$. El estudio se realizará con todos los datos obtenidos entre los años 2010 y 2014 de los formatos de informe de los accidentes laborales de los trabajadores sucedidos en el Hospital Antonio Lorena del Cusco.

Los datos fueron recolectados en un formato desarrollado en base a las variables que fueron estudiadas, se tuvo acceso a los informes de los accidentes laborales de los trabajadores desde 2010 al 2014 en la oficina de Epidemiología del hospital, previa solicitud de acceso. También se recabó información de la oficina de legajos, de la unidad de potencial humano, para elaborar los parámetros generales de los trabajadores del hospital Antonio Lorena.

La validación del instrumento: la hoja de recolección de datos se validó mediante juicio de tres expertos, dos son médicos especialistas Magísteres en Medicina Interna, y un experto médico especialista es Doctor en Medicina Interna. Se les entregó los formatos y el protocolo para que pudieran observar la coherencia y concordancia de lo que se pretendía conseguir con la hoja de recolección de datos con los objetivos y las hipótesis del trabajo de la investigación, los formatos de juicio de expertos fueron devueltos días después con su respectiva calificación. De la tabla de evaluación de expertos totalizando los tres evaluadores resulta bueno $3=11$, Muybueno 4=19; y de la ficha de validación de instrumentos bueno 41 a $60 \%=16$ y Muybueno 61 a $80 \%=14$.

Para el análisis de datos se utilizó estadística descriptiva para todas las variables, determinando la media, desviación estándar, para las variables cuantitativas y porcentajes o proporciones para las variables cualitativas; para correlacionar variables se usará la prueba de correlación de Spearman, debido a que en la prueba de normalidad de Kolmogorov Smirnov las distribuciones de las variables no son normales. Las variables fueron descargadas en el programa Excel y las estadísticas se realizaron en el programa SPSS 20. 


\section{RESULTADOS}

Los accidentes laborales reportados durante los años 2010 al 2014 en el hospital Antonio Lorena del Cusco fueron de 91 reportes. La edad promedio de los trabajadores lesionados es de 37,44 años con una desviación típica de 12,76 años con edad mínima de 19 años y una máxima de 65. El número total de trabajadores del Hospital Antonio Lorena es de 712 al 31 de diciembre del 2014, incluyendo a los internos de medicina, enfermería y de otros grupos ocupacionales, de los cuales 477 son mujeres $(66,99 \%)$ y 235 varones $(33,01)$, siendo el más joven de 21 años y el más antiguo de 70 años con un promedio de 47,35 años y desviación típica de 11,65 con una mediana de 49 años y una moda de 60 años.

Los profesionales con más accidentes laborales son los licenciados en enfermería con $34,1 \%$, seguido de los técnicos de enfermería con $23,1 \%$, luego los médicos con 13,2\%.En los grupos profesionales de medicina $\mathrm{y}$ enfermería los accidentes laborales son más frecuentes en el grupo de edad de menos de 29 años mientras que en los técnicos de enfermería los accidentes laborales son en el grupo de edad de 50 a 59 años de edad, siendo éstos grupos profesionales los que más accidentes laborales sufrieron (Tabla 1).

El tiempo de servicios promedio de los trabajadores con accidentes laborales es de 9,41 años con un mínimo de 15 días y un máximo de 34 años de servicios en la administración pública, El mayor número de accidentes se sucedió en los que trabajaron menos de 5 años con 59,3\% y un segundo repunte en el grupo de 27 a 30 años de servicios con 9,9\%. La hora promedio en que se produjeron los accidentes fue las 11 horas, las horas más frecuentes fueron las 08 y $09 \mathrm{hs}$ cada una con 9 accidentes; seguido de las $10 \mathrm{hs}$ ( 8 accidentes) y las 11 y 14 hs. con 7 accidentes.

En relación al género el sexo femenino es el más afectado con $84,6 \%(77$ accidentes) y el masculino con $15,4 \%(14$ accidentes). El nivel de educación alcanzado y los accidentes de trabajo se puede ver que $35,2 \%$ tienen un nivel de educación superior incompleto; el 30,8\% tiene superior técnico completo y en tercer lugar el 19,8\% nivel superior completo.

La forma como se sucedieron los accidentes tiene una distribución con el

Tabla 1. Tabla de contingencia categoría profesional edad del trabajador

\begin{tabular}{|c|c|c|c|c|c|c|}
\hline \multirow[t]{2}{*}{ Grupo Profesional } & \multicolumn{5}{|c|}{ Edad del Trabajador (agrupado) } & \multirow[t]{2}{*}{ Total } \\
\hline & $<=29$ & $30-39$ & $40-49$ & $50-59$ & $60-69$ & \\
\hline Medicina & 10 & 2 & 0 & 0 & 0 & 12 \\
\hline Enfermería & 16 & 7 & 2 & 5 & 1 & 31 \\
\hline Obstetricia & 0 & 1 & 2 & 0 & 0 & 3 \\
\hline Servicio Interno & 4 & 3 & 3 & 0 & 0 & 10 \\
\hline Técnico Administrativo & 0 & 0 & 2 & 3 & 0 & 5 \\
\hline Albañil & 0 & 0 & 1 & 0 & 0 & 1 \\
\hline Técnico de Lavandería & 0 & 0 & 1 & 0 & 0 & 1 \\
\hline Técnico Laboratorio & 3 & 0 & 1 & 0 & 0 & 4 \\
\hline Técnico Enfermería & 0 & 5 & 5 & 10 & 1 & 21 \\
\hline Técnico Nutrición & 0 & 0 & 1 & 2 & 0 & 3 \\
\hline Total & 33 & 18 & 18 & 20 & 2 & 91 \\
\hline
\end{tabular}

Elaboración propia 
primer lugar de los pinchazos con el $67,0 \%$ de los accidentes, en segundo lugar, caídas a nivel con $12,1 \%$ y luego contacto con líquidos con $6,6 \%$ de los accidentes. El agente causante de los accidentes laborales las agujas ocupan el primer lugar con $61.5 \%$ (56 casos), en segundo lugar el piso ocupa un $12,1 \%$ y en tercer lugar los líquidos en general con $6,6 \%$ (6 casos), en relación a lo anterior, la parte del cuerpo humano que más lesiones tubo fue la mano y los dedos de la mano alcanzando un 64.8\%(59 casos), en segundo lugar fueron los ojos 5.5\%(5 casos) y las rodillas en un $4.4 \%(4$ casos).

La correlación que se usó fue la de Spearman debido a que las variables no tienen una distribución normal y son ordinales; arrojó los siguientes resultados:

-El coeficiente de correlación entre la edad del trabajador y la forma del accidente laboral es de $-0,516$ y p menor de 0,001 .

-El coeficiente de correlación entre el tiempo de servicios del trabajador y la forma del accidente laboral es de $-0,490$ y p menor de 0,001 .

-El coeficiente de correlación entre el nivel de educación del trabajador y la forma del accidente es de $-0,086$ y $p=0,420$.

-El coeficiente de correlación entre la categoría profesional y la forma del accidente es $<0,369$ y p menor de 0,001 .

\section{DISCUSIÓN}

La edad promedio de los accidentes laborales en el Hospital Antonio Lorena del Cusco entre los años 2010 al 2014 es de 37,44 años, mucho menor que el promedio de edad de los trabajadores de la institución que fue de 47,35 años, lo que no indicaría que los accidentes laborales se producen en trabajadores más jóvenes. La edad de los lesionados no sigue una curva normal con una asimetría positiva que se encontraría en relación con la inexperiencia del trabajador; de igual manera el tiempo de servicios tiene una asimetría positiva por el mismo motivo mencionado. El coeficiente de correlación entre la edad del trabajador y la forma del accidente laboral es de $-0,516$ negativo y de mediana correlación.

El número de accidentes laborales reportados fueron de 91 reportes de 712 trabajadores durante cinco años que resulta mucho menor que los 299 casos reportados en el Hospital de Clínicas de Montevideo Uruguay en un periodo de tres años (11) y los 112 reportados en Albacete en dos años de 1023 trabajadores (4). Probablemente ésta diferencia se deba a la mayor complejidad del centro hospitalario de una capital de un país comparado con un hospital de provincia y tal vez porque en nuestro hospital no se reportan la totalidad de casos.

Se evidenció mediana correlación entre el tiempo de servicios y la forma del accidente rho -0.490 . El promedio del tiempo de servicios promedio 9,49 años de los accidentados mientras que el tiempo de servicios promedio del total de trabajadores es de 19,79 años, la mayor cantidad de los accidentes laborales es en la personas que tienen poco tiempo de servicios en los grupos profesionales que se encuentran en contacto directamente con el paciente como lo médicos, enfermeras, técnicos de enfermería y también los trabajadores de servicio interno, encargados de la limpieza del hospital, muy contrario a lo encontrado en el Hospital San Carlos de Madrid España cuyo promedio de edad de los accidentados fue de 61 años(6).

Las horas en que se produjeron más frecuentemente fueron la 08,09 y 10 horas, por la mañana, horas en las que se ingresa al trabajo, se inicia los 
tratamientos a los pacientes, se inicia los traslados a pacientes a diferentes unidades, se inicia trámites administrativos, etc. Semejante a lo encontrado en el Hospital Sabogal de Lima. En contraposición de los hallado en el Hospital de Agudos de España donde se reportaron la mayoría de los accidentes por la tarde, con el aumento de la edad, en cocina y lavandería y los diagnósticos más frecuentes tendinitis y esguinces.

77 accidentes se produjeron en mujeres, mientras que 14 en varones, semejante a lo hallado en Albacete España (5). Esto también es comprobado por contraste de la prueba del chi cuadrado ( $p$ menor a 0,001$)$ en relación a que la mayoría del personal de enfermería y técnicos de enfermería son mujeres y muy pocos varones. Esta situación es completamente inversa a los informes estadísticos del Ministerio del Trabajo del Perú donde el mayor porcentaje es en varones $(26,27)$. La frecuencia de producción de los accidentes laborales en personal de salud según la OPS es el tercero a nivel mundial (28).

El índice de correlación entre el grupo profesional y la forma del accidente es rho $=-0,369$, que es negativa $y$ moderada, pero tendiendo a baja. El personal de enfermería, técnicos de enfermería y medicina son los grupos ocupacionales que más reportaron accidentes laborales con $70,1 \%$ de los casos, semejante a lo encontrado en León España (4), Hospital de Clínicas Uruguay (11), pero contrario al Hospital de Agudos Dr. Peset Valencia España(7). Al realizar el contraste con chi cuadrado se observó una diferencia significativa en cuanto al grupo profesional de enfermería, técnicos de enfermería porque son ellos los que se encuentran directamente relacionados al tratamiento de los pacientes principalmente en el tratamiento endovenoso con agujas ( $\mathrm{p}$ menor a 0,001).
El coeficiente de correlación entre el nivel de educación del trabajador y la forma del accidente es de -0,086; prácticamente no hay correlación.

Los pinchazos por agujas son la primera causa de los accidentes laborales con $67 \%$ de los reportes, en segundo lugar las caídas a nivel 12,1\% y luego los contactos con líquidos $6,6 \%$, semejante a lo encontrado en Albacete (5), Uruguay (11), diferente a lo encontrado en Hospital de Agudo de Valencia en que fueron los esguinces y tendinitis (7). Los pinchazos son considerados como accidentes de discapacidad temporal leve cuyos procedimientos de tratamiento están bien establecidos $(29,30,31)$.

En concordancia con la forma del accidente producido por pinchazos, el segmento de cuerpo más frecuentemente lesionado es la mano y dedos de la mano $(68,4 \%)$ y los accidentes penetrantes $(61,5 \%)$, similar a lo reportado Albacete (5), Uruguay (11), al contraste con chi cuadrado nos refiere que estas diferencias no fueron debidas al azar(p menor a 0,001).

\section{CONCLUSIONES}

-Primero. La edad promedio de los accidentes laborales reportados es de 37,44 años siendo el grupo de edad con mayor cantidad de accidentes laborales los menores de 29 años 36,26\% (33 casos). La correlación entre la edad del trabajador y los accidentes laborales en de mediana correlación rho $-0,516 \mathrm{p}$ menor de 0,001.

- Segundo. No se pudo demostrar buena correlación entre el tiempo de servicios y la forma del accidente rho $-0,490 \mathrm{p}$ menor 0,001 .

- Tercero. El grupo ocupacional con mayor cantidad de accidentes laborales es enfermería con $34,01 \%$ (31 casos). Tampoco se pudo demostrar buena 
correlación entre grupo profesional y forma de accidente es mediana rho $-0,369$ p menor a 0,001 .

- Cuarto. No hay correlación entre el nivel de educación del trabajador y la forma del accidente rho $-0,086$ y $\mathrm{p}=$ 0,420 .

\section{REFERENCIAS}

(1) Aiken LH, Sloane DM, Klosinsky J. Hospital nurse's occupational exposures to blood: prospective, retrospective and institutional reports. Am. J. Public Health. 1997; Nro.87, p. 103-07.

2. International Institute for Occupational Safety and Health-NIOSH. Preventing needlestick in health care settings. Cincinnati (EUA). 1999.

(3) Haniharan A. Critical review of the literature on shays injuries. Journal of Advanced Nursing. 1997, 25 p.

144-54.

(4) López Gil j, De Luis Arribas L, Naveiro Rilo J et

al.

Accidentes de trabajo en personal relacionado con la sanidad en la provincia de León. Revista Sanidad Pública. Nro 1. España enero febrero 1994.

(5) Casares GabaldonI, Delicado Useros M, Paños de la Casa $\mathrm{T}$, et al. Accidentabilidad laboral en personal de enfermería en el Hospital General de Albacete. Escuela Universitaria de Enfermería de Albacete. Departamento de enfe rmería. Universidad de Castilla - La Mancha. 1993.

(6) Cavanillas Subero R, Cruzet Fernández F, Fereres Castiel J. Accidentes de trabajo del personal de un hospital general: evaluación de un método estudio epidemiológico basado en la entrevista personal con los accidentados. Servicio de Medicina Preventiva Hospital Universitario de San Carlos Madrid. Salud y Trabajo. España Nro. 611987.

(7) Pérez Bermúdez B, Tenias Murillo J, Tolosa Martínez $\mathrm{M}$, et al. Accidentes de trabajo en un hospital de agudos. Revista Española de Salud Pública. España

1998; 72: p 127 36.

(8) Consejo Profesional y Manejo de A ccidentes Laborales del Trabajador de Salud Expuesto a Virus de Transmisión Expuestos por Vía Hemática (VIH; hepatitis B y C).

(9) Palucci Marziale M, Carmo Cruz Robassi M.

Accidentes de trabajo con material cortopunzante en enfermeras de hospitales. Nure Investigation, Nro

2. Riberao Preto Brasil. Febrero 2004.

(10) Galindez L. Riesgos laborales de los trabajadores de salud. Salud de los Trabajadores. Versión impresa ISSN 1315 - 0138 v.15 n.2 Maracay dic. 2007. Universidad de Carabobo 2014.

(11) Tomasina F, Gómez Etchebarne F. Accidentes laborales en hospital de clínicas. Revista Médica Uruguay 2001; 17

$156-160$.
(12) Carvallo Suarez B. Una responsabilidad

compartida.

Revista de Enfermería. Colombia

2003.

(13) Nuñez Sarazu L, Ramirez Diaz R. Características epidemiológicas de los accidentes laborales punzocortantes y de exposición mucocutánea en el personal asistencial de enfermería del hospital

Alberto Sabo gal Sologuren Essalud . Revista

Científica de Enfermería. Recien p 37 -

42.

(14) Dirección de Salud Ocupacional - MINSA. Lima - Perú. Plan nacional de prevención de accidentes punzocortantes y exposición ocupacional a agentes patógenos de la sangre. Resumen del Primer Informe. Año 2009.

(15) MINSA. Directiva MINSA-DGSP-DEAISV.02 Sistema de manejo post exposición ocupacional al virus de la inmunodeficiencia humana en los trabajadores de salud.

(16) Coordinadora Interfederal de Salud, Confederación de Sindicatos de Ho landa. Manual para las comisiones de salud laboral (COSAL). Lima octubre del 2007.

(17) Congreso de la República del Perú. Ley 29783 Ley de Seguridad y Salud en el Trabajo. Julio del 2011.

(18) La Presidencia de la República del Perú. Reglamento de la Ley Nro. 29783 Ley de Seguridad y Salud en el Trabajo. Abril 2012.

(19) Avanto Belez W. Diseño y desarrollo del proyecto de investigación: guía de aprendizaje. Escuela de Post grado Universidad Cesar Vallejo. Trujillo - Perú -2014.

(20) Díaz Narvaez D. Metodología de la investigación científica y bioestadística : para médicos, odontólogos y estudiantes de ciencias de la salud. Primera Edición. Ril editores. Chile 2006

(21) Hernández Sampieri R, Fernández Collado C, Baptista Lucio P. Metodología de la investigación. Mc Graw Hill. Colombia 1997.

(22) Martínez González M. Bioestadística amigable, 2da Edición. Ed Diaz de Santos. España 2006.

(23) Chernick M. The essential of biostatistics for physicians, nurses and clinicians. Ed. Wlile y. Singapore 2011.

(24) Barton B, Peat J. Medical statistics. Second edition. Wiley editors. Reino Unido 2014.

(25) Estilo Vancouver. Biblioteca de la Universidad de Alicante. España.

(26) Ministerio de trabajo y promoción del empleo.

Boletín estadístico mensual de notificaciones de accidentes de trabajo, incidentes peligrosos y enfermedades ocupacionales. Año 1 N $\mathrm{N}^{\mathrm{RO}} 4$. Lima diciembre 2011.

(27) Ministerio de trabajo y promoción del empleo.

Boletín estadístico mensual de notificaciones de accidentes de trabajo, incidentes peligrosos

y enfermedades ocupacionales. Año 2 NRO12. Lima diciembre 2012. 
28.- Lovaine M, Ampliar y mantener la fuerza laboral de la salud mundial. OPS 2009.

(29) La Presidencia de la Republica España. Accidentes laborales y enfermedades profesionales: reglamento del decreto ley 18846. Decreto supremo $\mathrm{n}^{\circ}$

002-72-

TR.

(30) Lira Villavicencio M. Procedimiento de atención de accidentes de trabajo. Ministerio de Energía y Minas del Perú. [Lima 2013].

(31) Tennessee L, Padilla M. Salud y seguridad de los trabajadores del sector salud: manual para gerentes y administradores. OPS. C 2005 